\title{
Decreased fructose-1,6-bisphosphatase-2 expression promotes glycolysis and growth in gastric cancer cells
}

\author{
He Li ${ }^{1 \dagger}$, Juan Wang ${ }^{1 \dagger}$, Huiyu Xu ${ }^{1}$, Rui Xing ${ }^{1}$, Yuanming Pan ${ }^{1}$, Wenmei Li ${ }^{1}$, Jiantao Cui ${ }^{1}$, Hongbing Zhang ${ }^{2 *}$
} and Youyong Lu ${ }^{1 *}$

\begin{abstract}
Background: Increasing evidence suggests that cancer is a metabolic disease. Here, we investigated the potential role of fructose-1,6-bisphosphatase-2 (FBP2), the enzyme that catalyses the hydrolysis of fructose-1,6-bisphosphate to fructose-6-phosphate and inorganic phosphate in glucose metabolism, in gastric cancer (GC) development.

Results: Our data indicated that FBP2 was downregulated in GC tissues (86.2\%, 100/116), and absent or low FBP2 expression in $\mathrm{GC}$ tissues was correlated with poor survival of GC patients $(P=0.019)$. Conversely, ectopic expression of FBP2 in GC cells activated AMP-activated protein kinase (AMPK) signalling, inhibited the Akt-mTOR pathway, suppressed glucose metabolism, enhanced apoptosis, and reduced cell proliferation. Bisulphite genomic sequencing (BGS) in gastric cancer cell lines revealed that the FBP2 promoter region was densely methylated, and treatment of GC cells with the demethylation reagent, 5-aza-2-deoxycytidine (5-Aza), led to an increase in FBP2 expression. Importantly, forced expression of FBP2 abrogated tumour formation of these GC cells in nude mice. Conclusion: Our results indicate that FBP2 does negatively regulate cell growth, and reduced expression of FBP2 may contribute to carcinogenesis for GC. These findings suggest that restoration of FBP2 expression can be a promising strategy for the target therapy of GC.
\end{abstract}

Keywords: FBP2, Glycolysis, Gastric cancer, Cell growth, Prognosis

\section{Background}

Gastric cancer (GC), the second most common cause of cancer death worldwide [1], has long been thought to result from a combination of environmental factors and genetic alterations [2]. Activation of oncogenes and inactivation of tumour suppressor genes have all been implicated in gastric tumourigenesis [3,4]. However, increasing evidence in recent years indicates that cancer is a metabolic disease [5], in which cells have lost their normal checks on cell proliferation, resulting in

\footnotetext{
* Correspondence: hbzhang2006@gmail.com; youyonglu@hsc.pku.edu.cn ${ }^{\dagger}$ Equal contributors

${ }^{2}$ State Key Laboratory of Medical Molecular Biology, Department of Physiology and Pathophysiology, Institute of Basic Medical Sciences and School of Basic Medicine, Peking Union Medical College and Chinese Academy of Medical Sciences, Beijing 100005, China

'Laboratory of Molecular Oncology, Key Laboratory of Carcinogenesis and Translational Research (Ministry of Education), Peking University Cancer Hospital/Institute, Beijing 100142, China
}

excessive bioenergetic and biosynthetic needs [6]. To sustain such a high demand, cancer cells must alter their metabolism [7]. In the 1920s, Otto Warburg discovered that cancer cells preferentially rely on glycolysis instead of oxidative phosphorylation of glucose in normal cells regardless of the status of oxygen supply $[8,9]$. As a result, key enzymes in the glycolysis pathway are upregulated to exert this "Warburg effect" for cancer cell proliferation and tumourigenicity $[10,11]$.

Fructose-1,6-bisphosphatase (FBP) is one of the key enzymes in glucose metabolism. This enzyme catalyses the hydrolysis of fructose-1,6-bisphosphate to fructose6-phosphate and inorganic phosphate and exists as two isoenzymes in mammals: FBP1 and FBP2. FBP1 is recognised as the key enzyme of gluconeogenesis, and upregulated FBP1 has been shown to suppress cancer cell growth $[12,13]$; recent studies indicate that FBP2 participates in glycogen synthesis from carbohydrate precursors [14]. 
In this study, we have shown that reduced FBP2 expression was associated with poor clinical outcome in GC patients, while FBP2 upregulation led to inhibition of glucose metabolism, cell proliferation and tumourigenicity. Our findings suggested that FBP2 could be a promising biomarker for predicting the prognosis of GC patients and might provide a potential target for GC therapy.

\section{Results}

Decreased FBP2 expression in primary tumours is correlated with poor prognosis of GC patients

In our previous study, we used significance analysis of microarrays (SAM) and Bayesian analysis of gene expression levels (BAGEL) to analyse our raw microarray data to identify genes with altered expression in gastric cancer [15]. Among the genes in the glucose metabolism pathway, FBP2 was the most obviously decreased gene in GC samples compared with adjacent and normal tissues (Figure 1A).

To verify the decreased FBP2 expression in GC, the level of FBP2 was determined in both GC cell lines and tissue specimens. FBP2 mRNA was decreased in all 5 GC cell lines (Figure 1B), and downregulation of FBP2 protein was confirmed by Western blot analysis (Figure 1C). RT-PCR analysis represented that decreased FBP2 expression was detected in $88 \%(14 / 16)$ GC tissue specimens compared to adjacent normal tissues (Figure 1D). In addition, immunohistochemistry (IHC) performed in tissue microarrays confirmed that FBP2 protein expression was also decreased in GC tissues, with $86.2 \%$ (100/116) of tumour tissues having low FBP2 expression and only 42.7\% (47/110) of adjacent normal tissues having low FBP2 expression (Additional file 1: Figure S1, Table $1, P<0.001$ ).

Kaplan-Meier survival analysis showed that $78.8 \%$ $(41 / 52)$ of patients whose tumours exhibited low FBP2 expression had a poorer outcome than those with high FBP2 expression (Figure 2A, Table 2, $P=0.006$ ). More specifically, all the samples were divided into different stages of differentiation. For the patients with poorly differentiated GC samples, those patients with lowly FBP2-expressed samples had poorer outcomes than those with highly FBP2-expressed ones (Figure 2B, $P=0.042$ ). Univariate analysis indicated that other factors including tumour depth $(P=0.004)$, lymph node status $(P=0.006)$ and distant metastasis $(P=0.020)$ were also associated with poor survival (Table 2). Multivariate analysis revealed that FBP2 expression was an independent prognostic predictor of GC (Table 3, $P=0.019$ ) in conjunction with tumour depth (Table 3, $P=0.012$ ).

\section{FBP2 inhibits cell proliferation and tumourigenicity}

To investigate FBP2 function, the BGC823 cell line was transfected with pcDNA3.1-FBP2 plasmid and used as a cell model for both in vitro and in vivo studies.
Transfection with pcDNA3.1-FBP2 plasmid resulted in FBP2 overexpression compared with empty pcDNA3.1 plasmid (Figure 3A). The 3-[4,5-dimethylthiazol-2-yl]2,5-diphenyl tetrazolium bromide (MTT) assay revealed that FBP2 overexpression remarkably reduced cell proliferation in a time-dependent manner (Figure 3B). In addition, BGC823 cells with pcDNA3.1-FBP2 plasmid formed fewer and smaller colonies than mock transfected cells. More importantly, FBP2 overexpression inhibited the growth of BGC823 xenografts. The combined results from all the mice showed that the tumours formed by BGC823 cells with pcDNA3.1-FBP2 plasmid were much smaller and weighed less than those formed by mock transfected cells (Figure 3C-D). The clonal origin of BGC823 cells with pcDNA3.1-FBP2 plasmid in the tumours was confirmed by staining the cells with an antiFBP2 antibody (Figure 3E-F).

\section{FBP2 inhibits aerobic glycolysis through interference of Akt-mTOR pathway}

Since FBP2 plays a key role in glucose metabolism [14], the effects of FBP2 overexpression on ATP content and lactate concentration were examined. BGC823 cells with pcDNA3.1-FBP2 plasmid showed decreased levels of ATP and lactate than mock transfected cells (Figure 4A-B), which decreased the ATP/AMP ratio in these cells. As AMP-activated protein kinase (AMPK) acts as a sensor of cellular energy status and can be activated by a reduction of ATP/AMP ratio [16], the expression of AMPK and other proteins involved in the Akt-mTOR pathway were also examined. The amount of p-AMPK was increased in the BGC823 cells with pcDNA3.1-FBP2 plasmid. In contrast, the amounts of p-Akt and p-S6 were decreased compared with mock transfected cells, although total AMPK, Akt, and S6 expression remained the same (Figure 4C). These data indicated that FBP2 overexpression led to AMPK activation, and thus inhibited Akt-mTOR signalling.

\section{FBP2 induces apoptosis}

Since aerobic glycolysis is a protective strategy against reactive oxygen species (ROS) [17] and ROS induces mitochondrial apoptosis [18], the level of intracellular ROS in BGC823 cells with pcDNA3.1-FBP2 plasmid and the impact of FBP2-overexpression on apoptosis were examined. Intracellular ROS in BGC823 cells with pcDNA3.1-FBP2 plasmid was higher than that in mock transfected cells (Figure 5A). In addition, annexin V/PI staining showed that BGC823 cells with pcDNA3.1-FBP2 plasmid had an increased percentage of annexin $\mathrm{V}^{+} / \mathrm{PI}^{-}$ and annexin $\mathrm{V}^{+} / \mathrm{PI}^{+}$cells, representing early apoptotic cells and late apoptotic/necrotic cells [19], respectively, compared to the mock transfected cells (Figure 5B). Taken together, these data suggested that FBP2 overexpression 


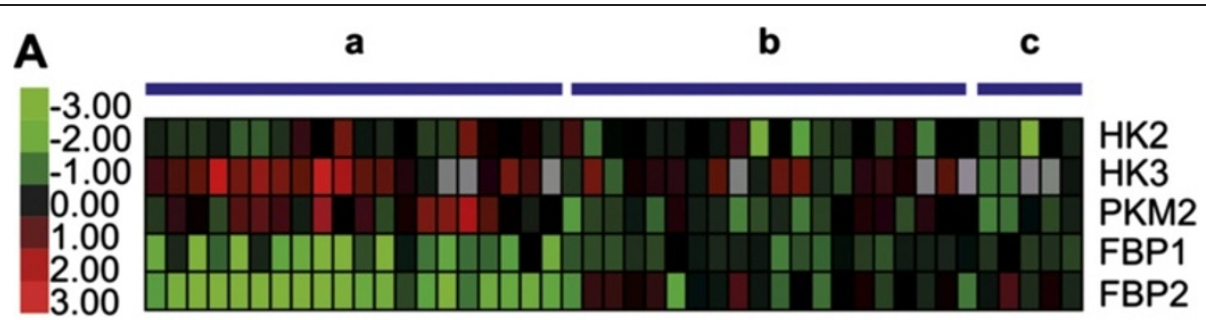

B
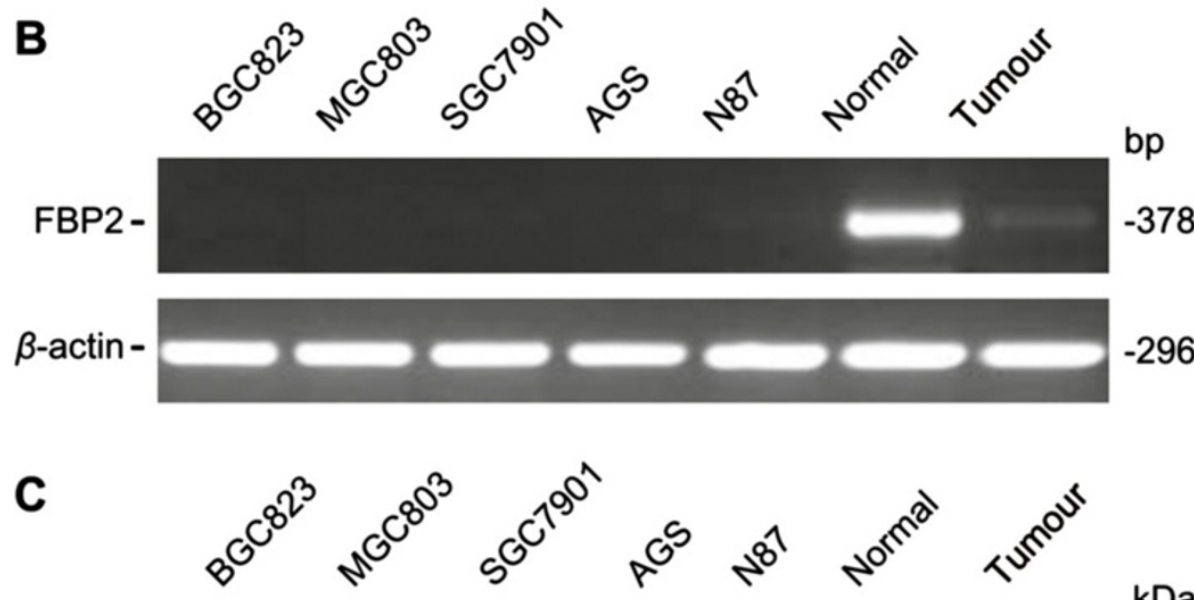

FBP2 -

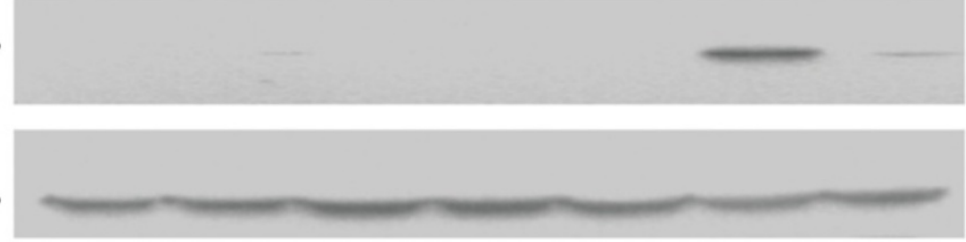

$\mathrm{kDa}$

$-37$

$\beta$-actin-

D

$$
\frac{1}{\mathrm{~N} T} \frac{2}{\mathrm{~N} T} \frac{3}{\mathrm{~N}} \frac{4}{\mathrm{~N} T} \frac{5}{\mathrm{~N} T} \frac{6}{\mathrm{~N} T} \frac{7}{\mathrm{~N}} \frac{8}{\mathrm{~N} T}
$$
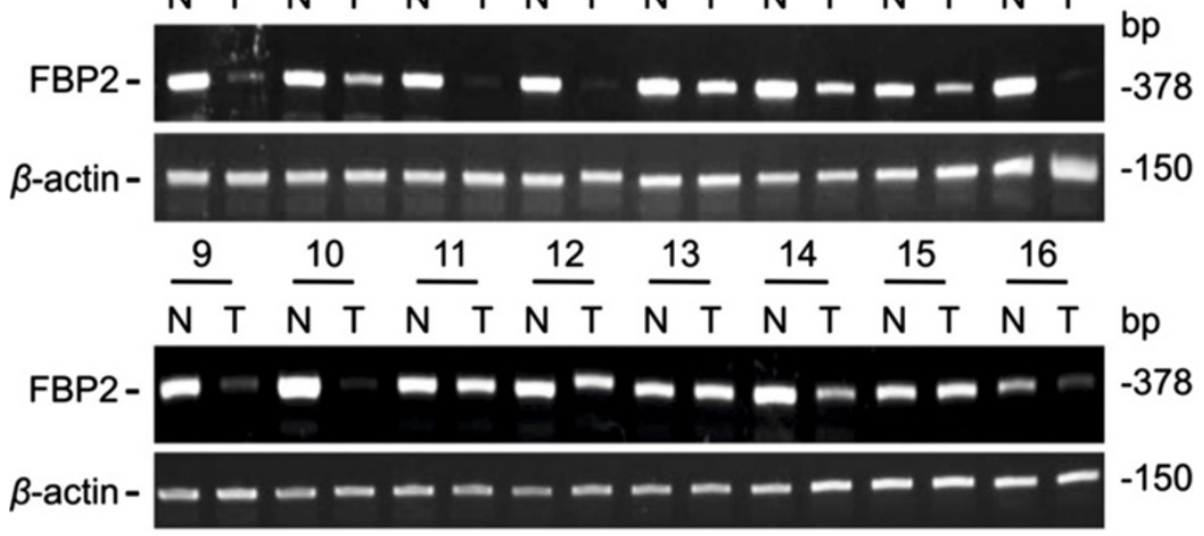

Figure 1 Decreased FBP2 in GC. (A) Glycolysis gene alterations were determined using Oligo gene microarray and high-qualified intestinal-type GC tissues between GC (20 cases) and paracancerous (20 cases) and normal gastric tissues (5 cases). Columns represent samples and rows represent genes; red colour represents high expression while green represents low expression. (a) GC versus common reference. (b) Adjacent normal cancer versus common reference. (c) Normal versus common reference. (B) Levels of FBP2 mRNA expression in GC cell lines BGC823, MGC803, SGC7901, AGS and N87 by RT-PCR. A pair of GC and adjacent normal tissues was used as controls. (C) Levels of FBP2 protein expression in GC cell lines BGC823, MGC803, SGC7901, AGS and N87 by Western blot analysis. A pair of GC and adjacent normal tissues was used as controls. (D) Levels of FBP2 mRNA expression in 16 pairs of GC (T) and adjacent normal tissues (N) were determined by RT-PCR. 
Table 1 Analysis of FBP2 expression in gastric tumours

\begin{tabular}{lcccc}
\hline & & \multicolumn{2}{c}{ FBP2 expression } & \\
\cline { 3 - 4 } Histology & Cases & High (\%) & Low (\%) & $P$ \\
\hline Normal & 110 & $63(57.3)$ & $47(42.7)$ & $<\mathbf{0 . 0 0 1}$ \\
Tumour & 116 & $16(13.8)$ & $100(86.2)$ & \\
\hline
\end{tabular}

Bold values indicate $P<0.05$.

led to a considerable increase in ROS in apoptotic cells compared to mock transfected cells. Protein profiling of the mitochondrial apoptotic pathway was performed to gain a deeper understanding of the underlying mechanism and revealed that the $\mathrm{Bax} / \mathrm{Bcl}-2$ ratio was increased and the activation of their downstream targets, caspase- 3 and caspase-9, were all induced in BGC823 cells with pcDNA3.1-FBP2 plasmid than mock transfected cells (Figure 5C).

\section{Hypermethylation of FBP2 promoter reduces the expression of FBP2 in GC}

Methylation status in the genomic FBP2 promoter sequence $(-2000$ to $+1000 \mathrm{bp})$ was investigated using the free online software, $\mathrm{CpG}$ Island Searcher $(\mathrm{GC}=55 \%$; ObsCpG/ExpCpG = 0.65; Length $=200$ bp; Gap between adjacent islands $=100$ bp) (Figure 6A). Two CpG islands FBP2-1 (-1338 to $-1014 \mathrm{bp})$ and FBP2-2 $(+29$ to $+229 \mathrm{bp}$ ) were identified and tested for their methylation status using Sequenom's MassARRAY system in the following GC cell lines: BGC823, MGC803, SGC7901, AGS, and N87. Substantially higher methylation was observed in the FBP2-2 region than in the FBP2-1 region, indicating that the $F B P 2-2$ region may be responsible for the low FBP2 expression observed in the GC specimens (Figure 6B). To confirm this finding, the methylation status of the FBP2-2 region was determined by bisulphite genomic sequencing (BGS). The FBP2 promoter was determined to be heavily methylated in BGC823,
MGC803, SGC7901, AGS and N87 cell lines using this method (Figure 6C). Importantly, FBP2 expression in these GC cell lines was substantially restored after treatment with the demethylating agent, 5-aza-2deoxycytidine (5-Aza) (Figure 6D). Furthermore, the FBP2 promoter was densely methylated in GC tissues compared to normal gastric tissues (Additional file 2: Figure S2).

\section{Discussion}

In the present study, we showed that decreased expression of $F B P 2$ gene in GC tissues correlated with poor patient survival. In addition, tumour depth serves as an important factor of survival for GC patients which shown in our observation. This finding indicated that the metabolic alterations increased the risk of death for GC patients. Conversely, overexpression of FBP2 suppressed glucose metabolism, cell proliferation, and induced apoptosis. Furthermore, CpG methylation of the FBP2 promoter was correlated with reduction of FBP2 expression, and FBP2 upregulation inhibited the Akt-mTOR and activated mitochondrial apoptotic pathways. Taken together, this inverse correlation between FBP2 expression and cell proliferation suggested that $F B P 2$ might act as a tumour suppressor gene in GC.

FBP helps to regulate the level of fructose-1,6bisphosphate, one of the most important intermediates in glycolysis, a major pathway generating ATP from glucose. Glycolysis metabolises glucose to pyruvate, which can then become the precursor of TCA cycle, the other major pathway directing from glucose to ATP. FBP converts fructose-1,6-bisphosphate to fructose-6-phosphate in the reverse direction of glycolysis [20]. Recent studies have shown that FBP1 overexpression could suppress glycolysis in cancer cells $[11,12]$. Stress that depletes cellular ATP can, in turn, activate energy sensor AMPK [16]. In our study,
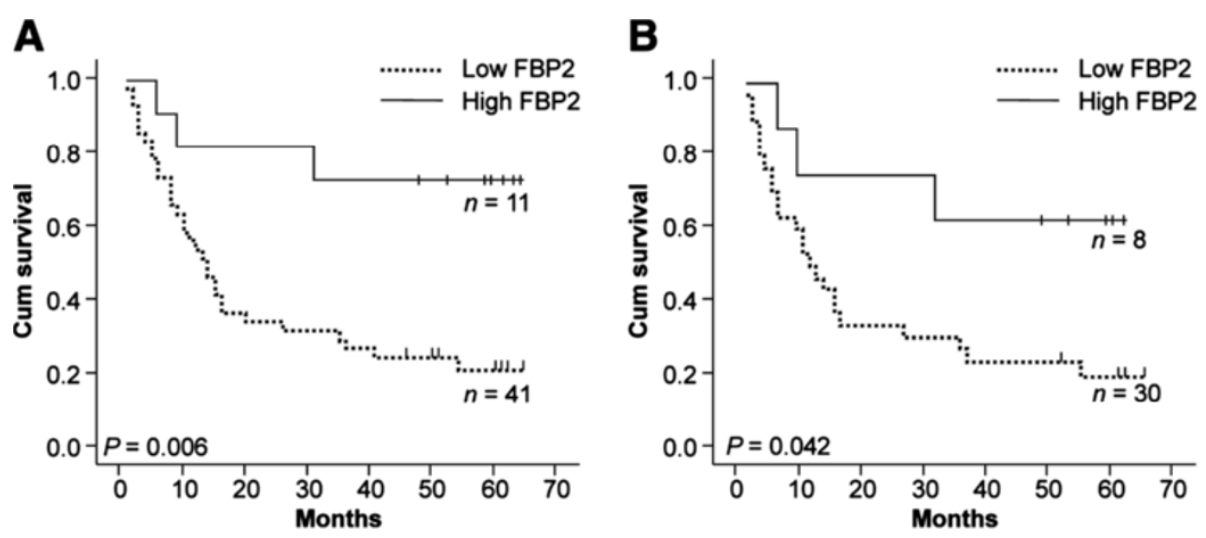

Figure 2 Kaplan-Meier plots of univariate analysis of overall survival of GC patients according to FBP2 expression of their tumours. (A) For all the patients, $(n=52, P=0.006)$. (B) For the patients with poorly differentiated sample, $(n=38, P=0.042)$. 
Table 2 Univariate analysis of overall survival

\begin{tabular}{|c|c|c|}
\hline Variable & Cases & $P$ \\
\hline \multicolumn{3}{|l|}{ Gender } \\
\hline Male & 36 & 0.625 \\
\hline Female & 16 & \\
\hline \multicolumn{3}{|l|}{ Age } \\
\hline$<60$ & 17 & 0.496 \\
\hline$\geq 60$ & 35 & \\
\hline \multicolumn{3}{|l|}{ Differentiation } \\
\hline Well/Moderate & 14 & 0.233 \\
\hline Poor & 38 & \\
\hline \multicolumn{3}{|l|}{ Tumour depth } \\
\hline $\mathrm{T} 1-\mathrm{T} 2$ & 12 & 0.004 \\
\hline T3-T4 & 40 & \\
\hline \multicolumn{3}{|l|}{ Lymph node status } \\
\hline NO & 18 & 0.006 \\
\hline N1-3 & 34 & \\
\hline \multicolumn{3}{|l|}{ Distant metastasis } \\
\hline MO & 42 & 0.020 \\
\hline M1 & 10 & \\
\hline \multicolumn{3}{|l|}{ FBP2 expression } \\
\hline High & 11 & 0.006 \\
\hline Low & 41 & \\
\hline
\end{tabular}

Bold values indicate $P<0.05$.

we found that the levels of ATP and lactate were significantly reduced after FBP2 overexpression, while p-AMPK expression was greatly increased (Figure 4), demonstrating that FBP2 overexpression suppresses energy production by aerobic glycolysis and TCA cycle.

In the present study, FBP2 not only suppressed the glycolysis pathway, but also cell proliferation in GC (Figure 3). Cancer cells face two major metabolic challenges: how to meet the bioenergetic and biosynthetic demands of abnormal proliferation. To overcome these challenges, cancer cells must reprogram their metabolism and become more dependent on aerobic glycolysis [6]. This allows the cancer cells to use the most abundant extracellular nutrient, glucose, to produce abundant ATP. Meanwhile, glucose degradation provides cells with intermediates needed for biosynthetic pathways [21]. These observations explain why the suppression of glycolysis by FBP2 led to inhibition of cell proliferation in GC cells.

The Akt-mTOR pathway, a master regulator of aerobic glycolysis and cellular biosynthesis, plays an important role in satisfying the bioenergetic and biosynthetic needs of cancer cells [21]. The role of Akt-mTOR in cellular energy metabolism is coupled to its function in cell proliferation, and mTOR appears to be the most critical
Table 3 Multivariate analysis of overall survival

\begin{tabular}{|c|c|c|}
\hline Variable & Cases & $P$ \\
\hline \multicolumn{3}{|l|}{ Gender } \\
\hline Male & 36 & 0.478 \\
\hline Female & 16 & \\
\hline \multicolumn{3}{|l|}{ Age } \\
\hline$<60$ & 17 & 0.698 \\
\hline$\geq 60$ & 35 & \\
\hline \multicolumn{3}{|l|}{ Differentiation } \\
\hline Well/Moderate & 14 & 0.214 \\
\hline Poor & 38 & \\
\hline \multicolumn{3}{|l|}{ Tumour depth } \\
\hline $\mathrm{T} 1-\mathrm{T} 2$ & 12 & 0.012 \\
\hline T3-T4 & 40 & \\
\hline \multicolumn{3}{|l|}{ Lymph node status } \\
\hline NO & 18 & 0.088 \\
\hline N1-3 & 34 & \\
\hline \multicolumn{3}{|l|}{ Distant metastasis } \\
\hline MO & 42 & 0.086 \\
\hline M1 & 10 & \\
\hline \multicolumn{3}{|l|}{ FBP2 expression } \\
\hline High & 11 & 0.019 \\
\hline Low & 41 & \\
\hline
\end{tabular}

Bold values indicate $P<0.05$

downstream effector of Akt required for cell proliferation and tumourigenicity [22]. Conversely, cellular energy status affects mTOR activity by the negative regulator AMPK [23], which at the same time, has an suppressing effect on Akt activity [24,25]. Thus, FBP2 upregulation in GC cells suppressed aerobic glycolysis, which in turn, inhibited the Akt-mTOR pathway by the negative regulator AMPK (Figure 4), thereby interfering with GC cell proliferation and tumourigenicity (Figure 3).

Since aerobic glycolysis is a protective strategy against ROS [17], the suppression of aerobic glycolysis could lead to an increase in ROS, which has been confirmed to play a role in the alteration of FBP1 [12]. Here, we showed that FBP2 overexpression increased ROS (Figure 5). Moreover, ROS can promote mitochondrial permeabilization and release of cytochrome $\mathrm{c}$, which can stimulate the caspase cascade (e.g. caspase-3, caspase-9), with pro-apoptotic protein Bax and anti-apoptotic protein $\mathrm{Bcl}-2$ as the major players in these pathways [18]. Thus, upregulation of FBP2 induced apoptosis in GC cells by increasing the Bax/Bcl-2 ratio and inducing caspase- 3 and caspase- 9 activation (Figure 5).

Akt is a major hub of cellular signalling, as its versatile functions can connect diverse upstream signalling pathways to even more diverse physiological outputs, such as 

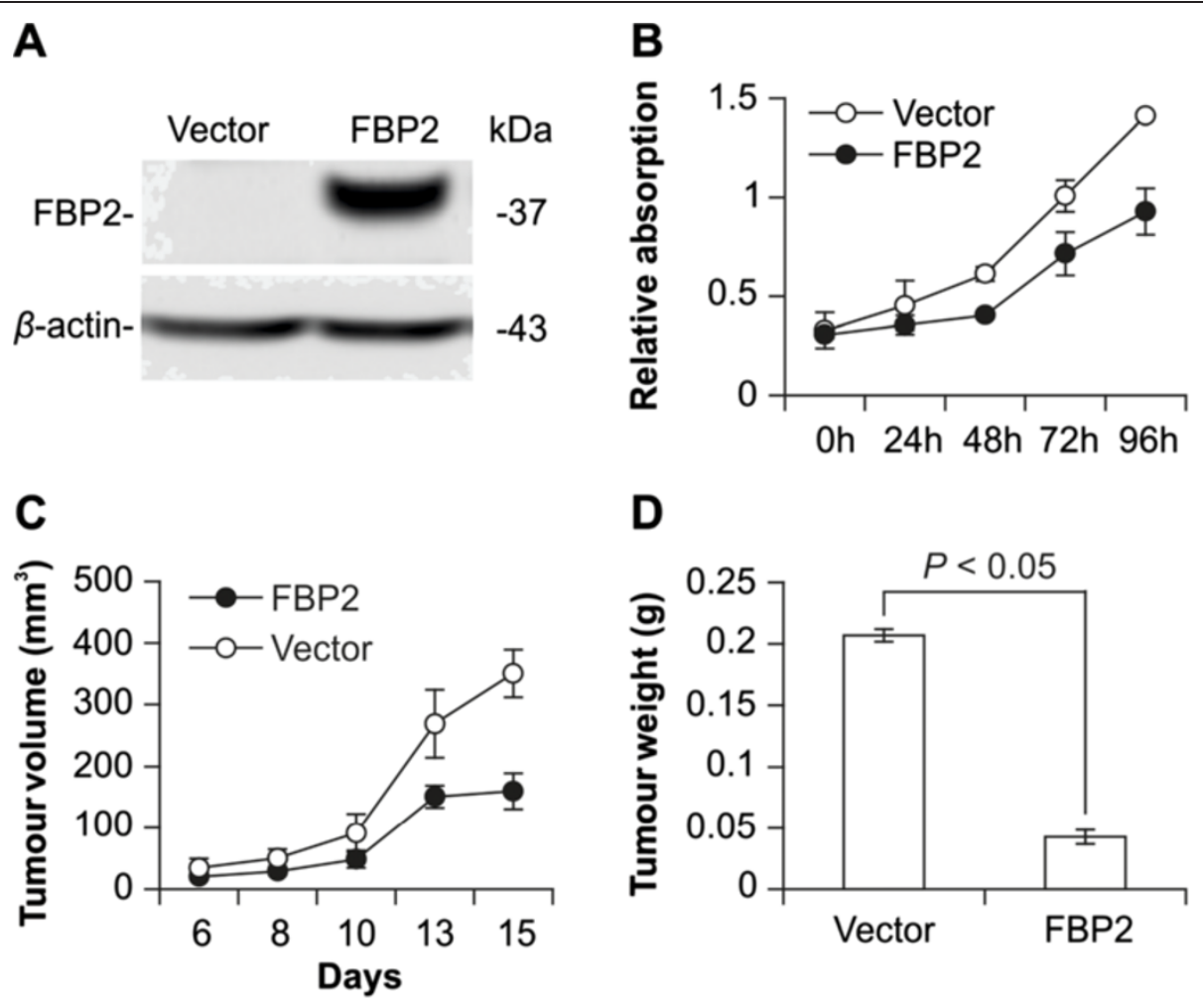

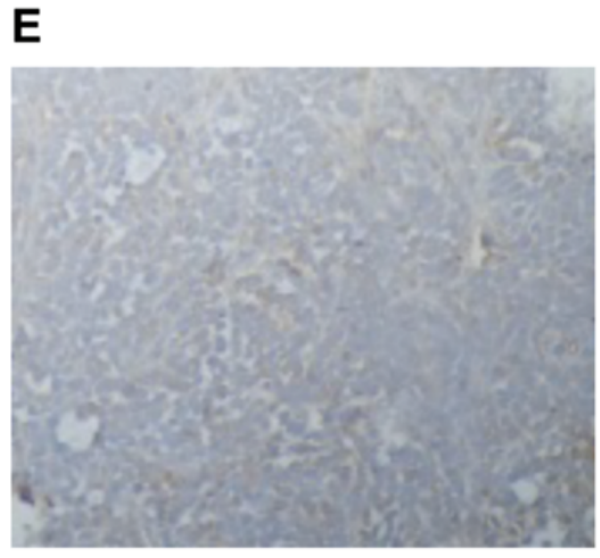

Vector
$\mathbf{F}$

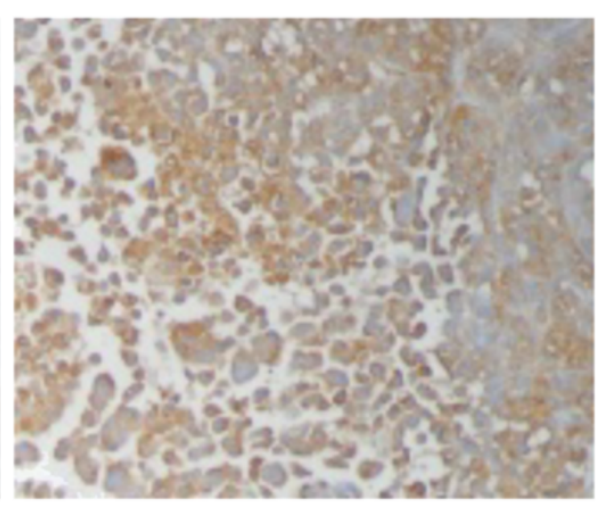

FBP2

Figure 3 Functional effects of FBP2 on cell proliferation and tumourigenicity. (A) Western blot analysis confirmed FBP2 overexpression in transfected BGC823 cells. (B) FBP2 overexpression suppressed cell proliferation in the MTT assay $(P<0.05)$. (C, D) FBP2-overexpressing BGC823 xenografts exhibited decreased tumour size and weight $(P<0.05)$. (E, F) $I H C$ staining showed FBP2 expression status in tumours $(200 \times)$.

promoting cell proliferation and inhibiting apoptosis [26]. In our study, a decrease in Akt could inhibit mTOR downstream targets, and in turn, cell proliferation. In contrast, decreased Akt could also enhance caspases activation in apoptosis. Therefore, FBP2 overexpression can simultaneously link Akt to the mTOR and apoptotic pathways.

Since methylation, one of the major epigenetic modifications of DNA capable of silencing gene transcription, plays an important role in cancer development [27-29], and contributes to the Warburg Effect [30], we also determined the methylation status of $F B P 2$ promoter. Our findings indicated that the $F B P 2$ promoter was highly methylated in GC (Figure 6), which has been confirmed in other types of cancer such as brain, liver, breast, and cervical cancers [31]. Thus, the decrease in FBP2 in GC might be correlated with promoter methylation and contribute to carcinogenesis in GC. 

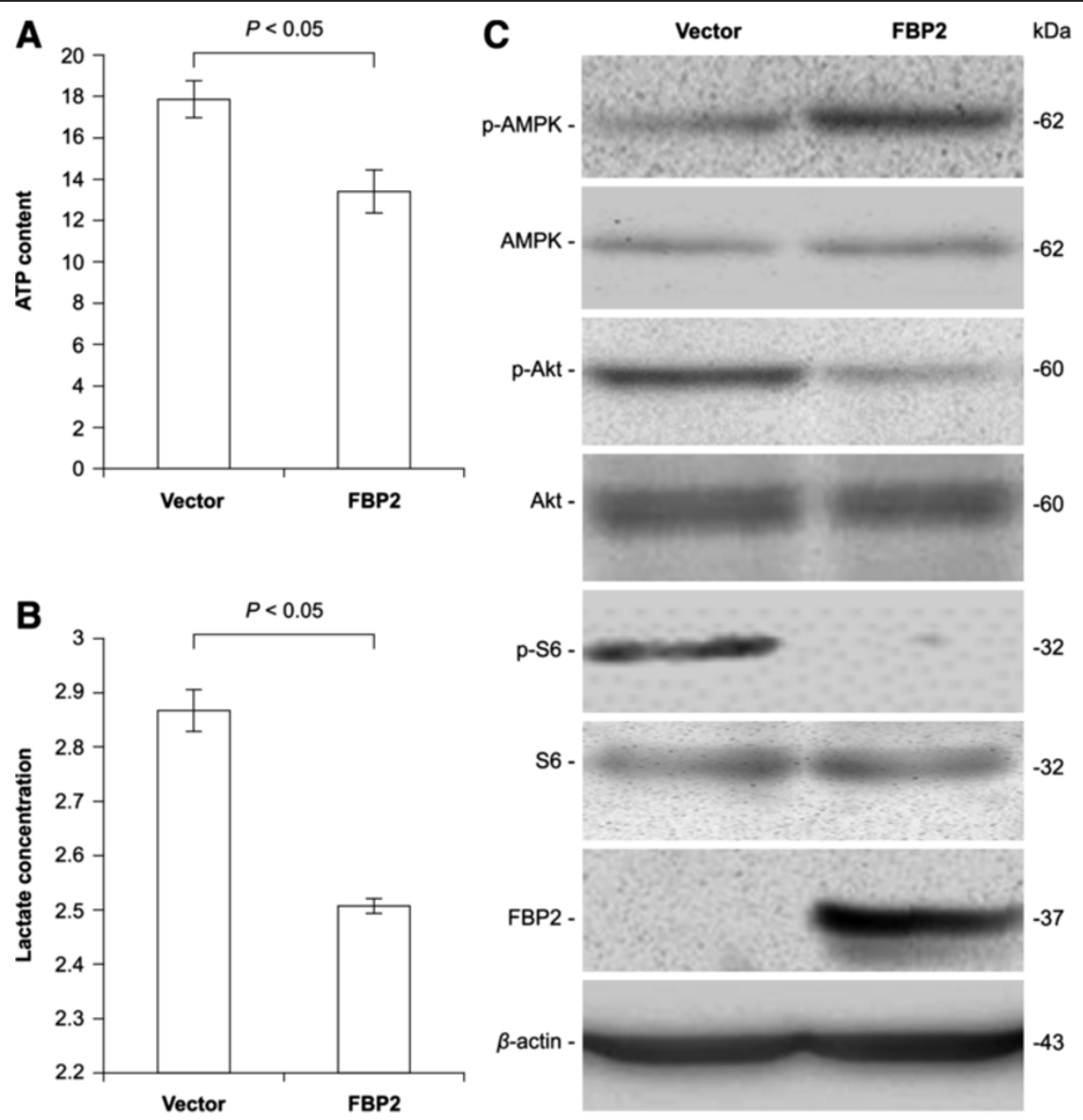

Figure 4 Functional effects of FBP2 on aerobic glycolysis. (A) Cellular ATP levels measured using a firefly luciferase-based ATP Assay Kit and normalised to controls showed that FBP2 overexpression inhibited ATP production $(P<0.05)$. (B) Lactate levels in cellular culture medium measured using a Lactic Acid Detection Kit and normalised to controls revealed that FBP2 overexpression inhibited lactate production $(P<0.05)$. (C) Western blot analysis showed inhibition of the Akt-mTOR pathway in BGC823 cells with pCDNA3.1-FBP2 plasmid compared to mock transfected cells,

\section{Conclusion}

FBP2 overexpression suppresses glucose metabolism and inhibits cell proliferation in GC, while reduced FBP2 expression contributes to cancer development. Taken together, restoration of FBP2 might be a novel and promising target for cancer therapy.

\section{Methods}

\section{Tissue specimens and IHC}

Sixteen specimens of primary GC and their paired noncancerous gastric mucosal tissues were collected from Beijing Cancer Hospital to determine the level of FBP2 mRNA expression. Another 11 specimens of primary GC and 6 normal gastric tissues were collected from Beijing Cancer Hospital to determine the methylation status of FBP2 promoter. A cohort of 116 formalin-fixed, paraffinembedded GC tissues was also obtained from Beijing Cancer Hospital, in which 52 cases had follow-up data.
This study was approved by the Institutional Ethical Standards Committee with the patients' written informed consents. The paraffin-embedded microarrays were stained with rabbit anti-FBP2 antibody (Abcam, Cambridge, MA, USA). IHC staining was performed using DAB (DAKO, Carpinteria, CA, USA).

\section{Cell culture}

Human GC cell lines BGC823, MGC803, and SGC7901 were established in China, and the AGS and N87 cell lines were purchased from ATCC (Manassas, VA, USA). All cells were cultured in Dulbecco's modified Eagle's medium (DMEM) with $5 \%$ foetal bovine serum and incubated in a humidified incubator $\left(5 \% \mathrm{CO}_{2}\right)$ at $37^{\circ} \mathrm{C}$. For demethylation treatment, cells were treated with $5 \mu \mathrm{M}$ 5-Aza (Sigma-Aldrich, St. Louis, MO, USA) for 72 h. Culture medium and drugs were replenished every $24 \mathrm{~h}$. 

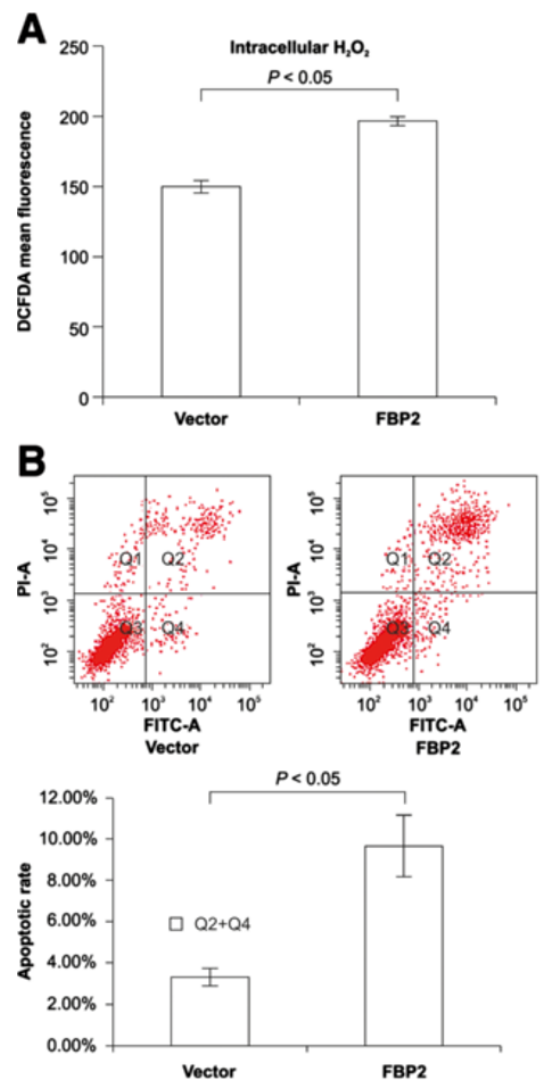

Figure 5 Functional effects of FBP2 on apoptosis. (A) FBP2 overexpression increased intracellular ROS determined using a ROS assay kit $(P<0.05)$. (B) AnnexinV/PI staining showed that FBP2 overexpression promoted apoptosis in BGC823 cells $(P<0.05)$. (C) Western blot analysis showed the effect of FBP2-upregulation on apoptotic-related genes in BGC823 cells.

\section{RNA extraction and RT-PCR}

Total RNA was extracted from cell lines and tissues using a RNApure total RNA isolation kit (Bioteke Corporation, Beijing, China). Reverse transcription reactions were carried out using EasyScript First-Strand cDNA Synthesis SuperMix (Transgen Biotech, Beijing, China), and semi quantitative RT-PCR was performed using $2 \times$ Taq PCR Mix (Genstar Biosolutions, Beijing, China). The primers used for amplification of FBP2 were 5'CAGGTTATGCGCTGTACGGT-3' (Forward) and 5'TGATGTAGGCCACGGGATTG-3' (Reverse). $\beta$-actin was adopted as an internal control in the RT-PCR reactions.

\section{Sequenom's MassARRAY EpiTYPER for methylation analysis}

The Sequenom MassARRAY platform (CapitalBio, Beijing, China) was used for quantitative analysis of FBP2 methylation. This system employs matrix-assisted laser desorption/ionization time-of-flight mass spectrometry in combination with RNA base-specific cleavage. The primer pairs for amplifying FBP2-1 (-1338 to $-1014 \mathrm{bp}$ ) and FBP2-2 $(+29$ to +229 bp $)$ regions were $5^{\prime}-$

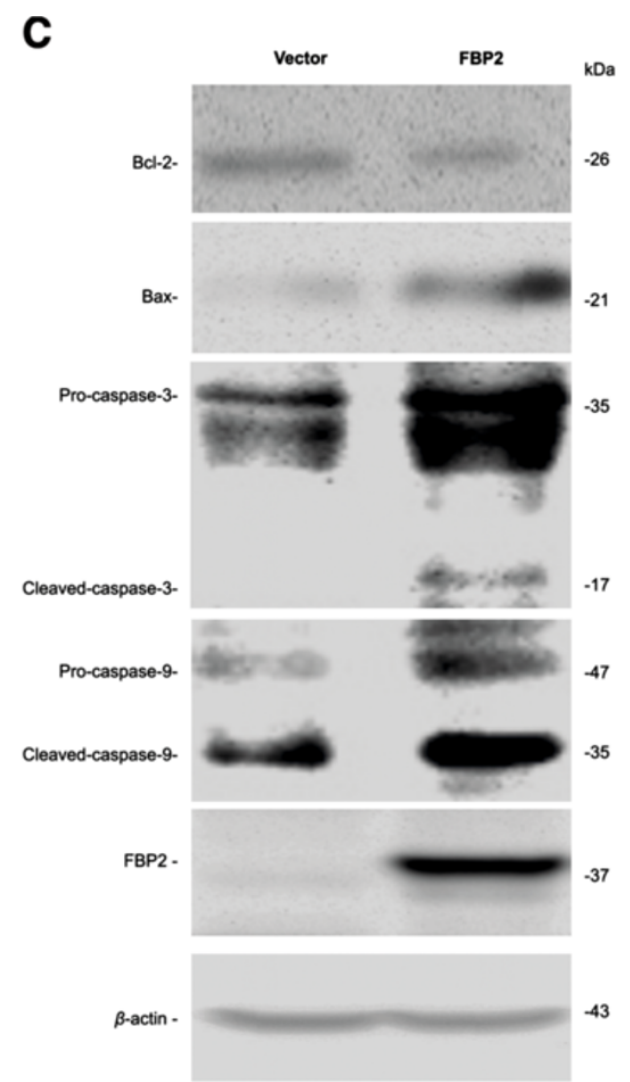

aggaagagagTTTTTGAGATGGAGTTTTATTTTGT-3' (FBP2-1 Forward) and 5' -cagtaatacgactcactatagggagaaggct CTCACGCCTATAATCCCAACAC-3' (FBP2-1 Reverse) and $5^{\prime}$-aggaagagagGAGATTTTGGTAGGGTTAGTATA GATT-3' (FBP2-2 Forward) and 5'-cagtaatacgactcactatag ggagaaggctAAAACTCACAAATAAACCAAACC-3' (FBP22 Reverse). Methylation ratios were generated using EpiTYPER software version 1.0 (Sequenom, San Diego, CA, USA).

\section{DNA extraction and methylation analysis}

Genomic DNA was extracted from cell lines and tissues using the genomic DNA rapid extraction kit (Aurora Biomed, Inc., Vancouver, B.C., Canada). Bisulphite modification of genomic DNA was performed using Zymo DNA Methylation Kit (Zymo Research, Irvine, CA, USA). Methylation status of FBP2-2 region was determined by methylation-specific PCR (MSP) and bisulphite genomic sequencing (BGS). MSP was carried out for 40 cycles with annealing temperature at $61^{\circ} \mathrm{C}$. Methylation-specific primers were: MF $5^{\prime}$-ACGGATAGAAGTTTTAGTTC GAAATC-3' and MR 5' ${ }^{\prime}$-AATAAACCAAACCGACCT TACG $-3^{\prime}$, and unmethylation-specific primers were: 
A

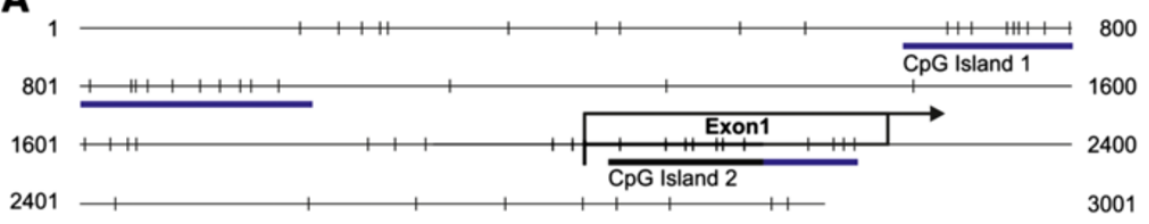

Select lower limits: $\% G C=55$, ObsCpG/ExpG $=0.65$, Length $=200$, Distance $=100$ CpG Island 1 start $=663$, end $=987, \% G C=56.3$, ObsCpG/ExpCG $=0.744$, Length $=325$ CpG Island 2 sart $=2030$, end $=2230 \% G C=57.7$, ObsCpG/ExpCG $=0.657$, Length $=201$

B

$0 \%$ - - 0 -0-0 100\%

FBP2-1

SGC7901

FBP2-2

SGC7901

C

BGC823

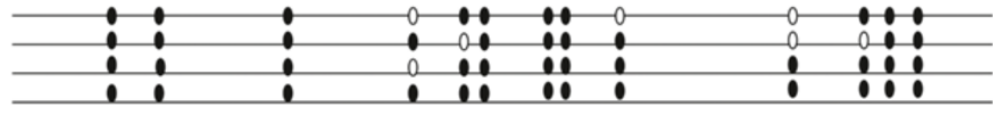

MGC803

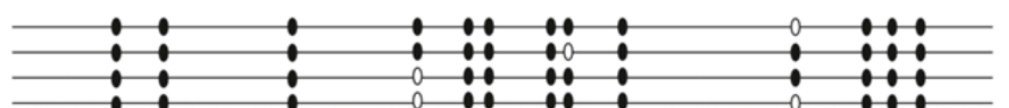

SGC7901

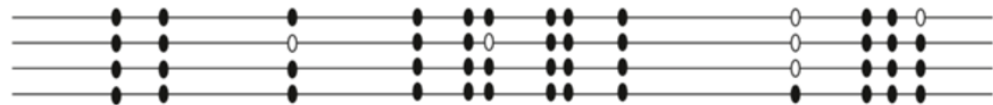

AGS

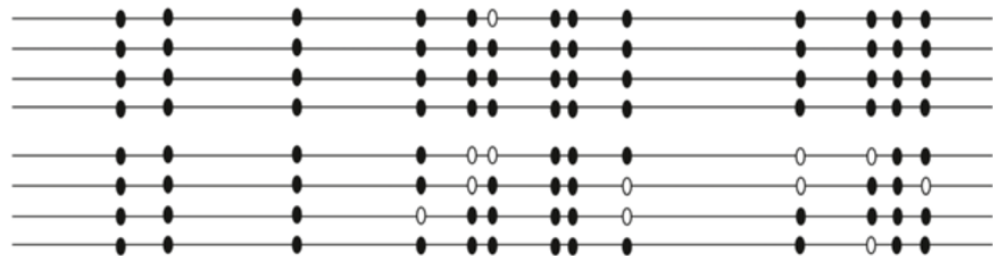

D

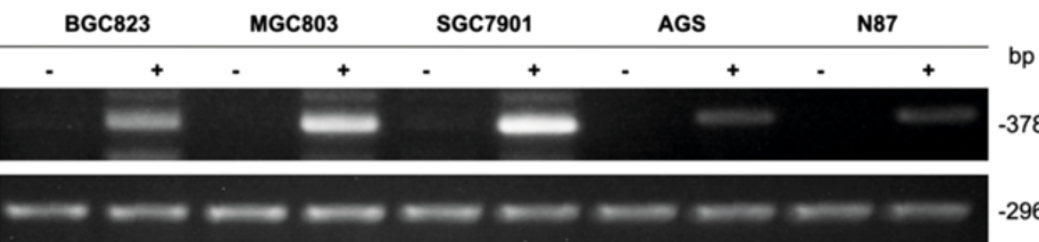

Figure 6 (See legend on next page.) 
(See figure on previous page.)

Figure 6 Decrease FBP2 in GC cells correlated with promoter methylation. (A) Schematic structure of FBP2 CpG islands around exon 1 $(+1$ to +237). (B) Profiling of site-specific methylation of FBP2 CpG islands: FBP2-1 and FBP2-2 in 5 GC cell lines: BGC823, MGC803, SGC7901, AGS, and N87. Different colours of circles mark the position of $\mathrm{CpG}$ dinucleotides within the sequence (straight line) and the levels of methylation. Gray circles represent the unanalyzed CpG sites. The "not analyzed" CpG sites have low mass or high mass which cannot be reliably detected. Data represent at least three independent experiments. (C) Methylation mapping of 13 CpG sites of the FBP2-2 CpG island obtained from BGS in BGC823, MGC803, SGC7901, AGS, and N87 GC cell lines. White and black circles represent unmethylated and methylated CpG sites, respectively. (D) Upregulation of FBP2 mRNA expression following treatment with 5-Aza in GC cell lines.

UF $\quad$ 5'-TGGATAGAAGTTTTTTTGAAATTGA-3' and UR 5'-CAAATAAACCAAACCAACCTTACAC-3'. For BGS, the following primers were used: BF $5^{\prime}$ GAGATTTTGGTAGGGTTAGTATAGATT-3' and BR 5' -AAAACTCACAAATAAACCAAACC-3'. The fragment amplified corresponded to the FBP2 promoter region, +29 and $+229 \mathrm{bp}$, and the ATG start codon of FBP2 was defined as +1 . PCR products were inserted into pEASY-T1 Cloning Vector (Transgen Biotech), and at least four colonies were randomly selected for sequencing.

\section{Ectopic expression of FBP2, cell proliferation and tumour formation}

Full-length FBP2 cDNA was cloned into pcDNA3.1 (Invitrogen, Grand Island, NY, USA) to construct the transferring plasmid, pcDNA3.1-FBP2. BGC823 cells were then transfected with either pcDNA3.1-FBP2 or empty pcDNA3.1 plasmid using the Lipofectamine 2000 reagent (Invitrogen) and underwent G418 (400 g/mL) selection to form stable colonies. Restoration of FBP2 expression was confirmed by Western blot analysis before these cells were used in any subsequent assays.

Cell proliferation of FBP2-overexpressing BGC823 was assessed using several different assays. For the MTT assay, BGC823 cells with pcDNA3.1-FBP2 or empty pcDNA3.1 plasmid were seeded into 96-well plates, and absorbance at $570 \mathrm{~nm}$ was recorded every $24 \mathrm{~h}$ for 5 days. For the soft agar assay, BGC823 cells with pcDNA3.1-FBP2 or empty pcDNA3.1 plasmid were suspended in DMEM with $0.3 \%$ agarose overlying $0.6 \%$ agarose, and colonies were stained with $0.2 \%$ INT after 3-4 weeks of culture.

To determine tumour growth in vivo, approximately $5 \times 10^{5}$ BGC-823 cells with pcDNA3.1-FBP2 or empty pcDNA3.1 plasmid were injected into female BALB/c nude mice, which were monitored periodically and sacrificed 3 weeks later. Tumour specimens from nude mice were weighed and immunostained. All manipulations involving live mice were approved by the Ethics Committee of Beijing Cancer Hospital/Institute.

\section{Measurement of ATP and lactate}

To measure ATP content and lactate concentration, a total of $2 \times 10^{5}$ cells per well were seeded into 12 -well plates for 1 day. Cell numbers were determined using a
Countess $^{\mathrm{TM}}$ automated cell counter (Invitrogen). Medium was collected to measure lactate levels Nanjing City, China). Lactate levels were determined by recording the absorbance at $530 \mathrm{~nm}$ using an Ultraspec 3300 Pro (GE Healthcare Bio-sciences Corp, New Jersey, USA), and data were normalised to the control group. Trypsindispersed cells were collected to measure the cellular ATP levels using a firefly luciferase-based ATP Assay Kit (Beyotime, Haimen City, China). Luminescence in the ATP assay was measured using an LMax II luminometre (Molecular Devices, Sunnyvale, CA, USA) and analysed by SoftMax Pro software Version 5 (Molecular Devices). Data were normalised to the control group.

\section{Measurement of intracellular ROS and annexin V/PI staining}

Intracellular ROS was examined using a ROS assay kit (Applygen, Beijing, China), and fluorescence was measured using a BD FACSAria flow cytometer (BD Biosciences, San Jose, CA, USA) ( $\lambda$ ex at $488 \mathrm{~nm}$ and $\lambda$ em at $520 \mathrm{~nm})$. Apoptosis-mediated cell death was examined using the Annexin V-FITC Apoptosis Detection Kit (Beijing Biosea Biotechnology Co. Ltd., Beijing, China). Cells were detected by flow cytometry (BD FACSAria), and data were analysed using FASCDiva software. Cells in early stages of apoptosis were annexin $\mathrm{V}$ positive and PI negative, whereas cells in the late stages of apoptosis were both annexin V and PI positive.

\section{Western blot analysis}

Total proteins were extracted from 5 GC cell lines in lysis buffer containing $50 \mathrm{mM}$ Tris (pH 6.8), $100 \mathrm{mM}$ DTT, 1\% SDS and 25\% glycerol. Equal amount of proteins were electrophoresed on 12\% SDS-PAGE and transferred to PVDF membranes using the Mini PROTEAN 3 system (BIO-RAD, Hercules, CA, USA). PVDF membranes were blocked in 5\% BSA and incubated with anti-FBP2 (1:1000, Abcam), anti-p-AMPK (1:500, Cell Signalling Technology, Danvers, MA, USA), anti-AMPK (1:500, Cell Signalling Technology), anti-p-Akt (1:500, Cell Signalling Technology), anti-Akt (1:500, Santa Cruz Biotechnology, Inc., Santa Cruz, CA, USA), anti-p-S6 (1:500, Epitomics, Burlingame, CA, USA), anti-S6 (1:500, Cell Signalling Technology), anti-Bcl-2 (1:500, US Biological, Boston, MA, USA), anti-Bax (1:500, Epitomics), 
anti-caspase-3 (1:250, Bioworld, St. Louis Park, MN, USA), or anti-caspase-9 (1:250, Bioworld) antibody, followed by the appropriate horseradish peroxidase-linked secondary antibody purchased from Proteintech Group Inc. (Chicago, IL, USA). Anti- $\beta$-actin (1:10000, Sigma) antibody was used to detect the loading control.

\section{Statistical analysis}

All statistical analyses were performed using SPSS 13.0 software. Student's t test was used to compare groups in the MTT and soft agar assays. Student's $t$ test was also used to determine remarkable differences between groups regarding tumour size and weight, ATP content and lactate concentration, ROS and apoptotic rate. The $x^{2}$ and Fisher's exact test were used to determine the difference of FBP2 protein expression levels between GC and adjacent normal tissues. The Kaplan-Meier method was used for plotting survival curves, and survival data were evaluated using multivariate Cox regression analysis. For all analyses, data were shown as mean $\pm \mathrm{SD}$, and $P<0.05$ was considered statistically significant.

\section{Additional files}

Additional file 1: Figure S1. Representative immunohistochemical staining of FBP2 in tissue microarrays (original magnification $\times 200$ ). (A) Normal gastric tissue. (B) The cervical part of normal gastric tissue. (C) Well/moderately differentiated GC tissue with high FBP2 expression. (D) Well/moderately differentiated GC tissue with low FBP2 expression. (E) Poorly differentiated GC tissue with high FBP2 expression. (F) Poorly differentiated GC tissue with low FBP2 expression. (G) A transition staining of FBP2 from GC to adjacent normal tissue.

Additional file 2: Figure S2. The methylation status of FBP2 promoter was determined by MSP and USP (unmethylation-specific PCR) in GC (T) and normal gastric tissues (N). Primer efficiency was verified by positive control (in vitro methylated DNA, IVD) and negative control (normal lymphocyte $\mathrm{DNA}, \mathrm{NL}$ ). $d d \mathrm{H}_{2} \mathrm{O}$, double-distilled water, was used as blank control. M, methylated alleles; $U$, unmethylated alleles.

\section{Abbreviations \\ GC: Gastric cancer; FBP: Fructose-1,6-bisphosphatase; \\ IHC: Immunohistochemistry; DMEM: Dulbecco's modified Eagle's medium; 5-Aza: 5-aza-2-deoxycytidine; MSP: Methylation-specific PCR; BGS: Bisulphite genomic sequencing; MTT: 3-[4,5-dimethylthiazol-2-yl]-2,5-diphenyl tetrazolium bromide; ROS: Reactive oxygen species; AMPK: AMP-activated protein kinase.}

\section{Competing interests}

The authors declare that they have no competing interests.

\section{Authors' contributions}

$\mathrm{HL}$ conceived the study, carried out the experiments, analysed data and drafted the manuscript; JW, HYX, YMP, WML and JTC carried out the experiments; RX conceived the study and analysed data; HBZ reviewed the manuscript and supervised the study; and YYL conceived and supervised the study, analysed data and finalised the manuscript. All authors read and approved the final manuscript.

\section{Acknowledgements}

This work was supported by grants from the National Bio-Tech 863 (2006AA02A402 and 2012AA02A504).
Received: 29 March 2013 Accepted: 13 September 2013

Published: 25 September 2013

\section{References}

1. Roder DM: The epidemiology of gastric cancer. Gastric Cancer 2002, 5:5-11.

2. Milne AN, Carneiro F, O'Morain C, Offerhaus GJA: Nature meets nurture: molecular genetics of gastric cancer. Hum Genet 2009, 126:615-628.

3. Zheng L, Wang L, Ajani J, Xie K: Molecular basis of gastric cancer development and progression. Gastric Cancer 2004, 7:61-77.

4. Peek RM, Crabtree JE: Helicobacter infection and gastric neoplasia. J Pathol 2006, 208:233-248.

5. Seyfried TN, Shelton LM: Cancer as a metabolic disease. Nutr Metab 2010, 7:7.

6. Jones $R G$, Thompson CB: Tumor suppressors and cell metabolism: a recipe for cancer growth. Gene Dev 2009, 23:537-548.

7. DeBerardinis RJ, Sayed N, Ditsworth D, Thompson CB: Brick by brick: metabolism and tumor cell growth. Curr Opin Genet Dev 2008, 18:54-61.

8. Warburg O: Über den stoffwechsel der carcinomzelle. Naturwissenschaften 1924, 12:1131-1137.

9. Warburg O: On the origin of cancer cells. Science 1956, 123:309-314.

10. Kroemer G, Pouyssegur J: Tumor cell metabolism: cancer's Achilles' heel. Cancer Cell 2008, 13:472-482.

11. Vander Heiden MG, Cantley LC, Thompson CB: Understanding the Warburg effect: the metabolic requirements of cell proliferation. Science 2009, 324:1029-1033.

12. Chen M, Zhang J, Li N, Qian Z, Zhu M, Li Q, Zheng J, Wang X, Shi G: Promoter hypermethylation mediated downregulation of FBP1 in human hepatocellular carcinoma and colon cancer. PLoS One 2011, 6:e25564.

13. Liu X, Wang $X$, Zhang J, Lam EKY, Shin VY, Cheng ASL, Yu J, Chan FKL, Sung $J J Y$, Jin HC: Warburg effect revisited: an epigenetic link between glycolysis and gastric carcinogenesis. Oncogene 2009, 29:442-450

14. Dzugaj A: Localization and regulation of muscle fructose-1,6bisphosphatase, the key enzyme of glyconeogenesis. Adv Enzyme Regul 2006, 46:51-71.

15. Zang S, Guo R, Zhang L, Lu Y: Integration of statistical inference methods and a novel control measure to improve sensitivity and specificity of data analysis in expression profiling studies. J Biomed Inform 2007, 40:552-560

16. Hardie DG: The AMP-activated protein kinase pathway - new players upstream and downstream. J Cell Sci 2004, 117:5479-5487.

17. Brand KA, Hermfisse U: Aerobic glycolysis by proliferating cells: a protective strategy against reactive oxygen species. FASEB J 1997, 11:388-395

18. Circu ML, Aw TY: Reactive oxygen species, cellular redox systems, and apoptosis. Free Radical Bio Med 2010, 48:749-762.

19. Vermes I, Haanen C, Steffens-Nakken H, Reutellingsperger C: A novel assay for apoptosis flow cytometric detection of phosphatidylserine expression on early apoptotic cells using fluorescein labelled Annexin V. $J$ Immunol Methods 1995, 184:39-51.

20. Berg JM, Tymoczko JL, Stryer L: Biochemistry. 6th edition. New York: W. H. Freeman; 2007.

21. DeBerardinis RJ, Lum JJ, Hatzivassiliou G, Thompson CB: The biology of cancer: metabolic reprogramming fuels cell growth and proliferation. Cell Metab 2008, 7:11-20.

22. Bhaskar PT, Hay N: The two TORCs and Akt. Dev Cell 2007, 12:487-502.

23. Hay N, Sonenberg N: Upstream and downstream of mTOR. Gene Dev 2004, 18:1926-1945

24. Priebe A, Tan L, Wahl H, Kueck A, He G, Kwok R, Opipari A, Liu JR: Glucose deprivation activates AMPK and induces cell death through modulation of Akt in ovarian cancer cells. Gynecol Oncol 2011, 122:389-395.

25. Rattan R, Giri S, Singh AK, Singh I: 5-Aminoimidazole-4-carboxamide-1- $\beta$ D-ribofuranoside inhibits cancer cell proliferation in vitro and in vivo via AMP-activated protein kinase. J Biol Chem 2005, 280:39582-39593.

26. Kim D, Chung J: Akt: versatile mediator of cell survival and beyond. J Biochem Mol Biol 2002, 35:106-115.

27. Jones PA, Baylin SB: The fundamental role of epigenetic events in cancer. Nat Rev Genet 2002, 3:415-428.

28. Esteller M: Cancer, epigenetics and the Nobel Prizes. Mol Oncol 2012, 6:565-566. 
29. Suzuki H, Maruyama R, Yamamoto E, Kai M: DNA methylation and microRNA dysregulation in cancer. Mol Oncol 2012, 6:567-578.

30. Wang $X$, Jin H: The epigenetic basis of the Warburg effect. Epigenetics 2010, 5:566-568

31. Bigl M, Jandrig B, Horn L-C, Eschrich K: Aberrant methylation of human L- and M-fructose 1,6-bisphosphatase genes in cancer. Biochem Bioph Res Co 2008, 377:720-724.

doi:10.1186/1476-4598-12-110

Cite this article as: Li et al.: Decreased fructose-1,6-bisphosphatase-2 expression promotes glycolysis and growth in gastric cancer cells. Molecular Cancer 2013 12:110

\section{Submit your next manuscript to BioMed Central and take full advantage of:}

- Convenient online submission

- Thorough peer review

- No space constraints or color figure charges

- Immediate publication on acceptance

- Inclusion in PubMed, CAS, Scopus and Google Scholar

- Research which is freely available for redistribution 